\title{
Structure and 3D-3D Topotactic Transformation of the Aluminophosphate Molecular Sieve PST-5 and Its Implication in New Zeolite Structure Generation
}

Zhehao Huang, ${ }^{1+}$ Seungwan Seo, ${ }^{2+}$ Jiho Shin, ${ }^{3}$ Bin Wang, ${ }^{1}$ Robert G. Bell, ${ }^{4}$ Suk Bong Hong ${ }^{2 *}$ and Xiaodong Zou ${ }^{1 *}$

${ }^{1}$ Bezerlii Center EXSELENT on Porous Materials, Department of Materials and Environmental Chemistry, Stockholm University, SE-106 91 Stockholm, Sweden

${ }^{2}$ Center for Ordered Nanoporous Materials Synthesis, Division of Environmental Science and Engineering, POSTECH, Pohang 37673, Korea

${ }^{3}$ Research Center for Convergent Chemical Process, Korea Research Institute of Chemical Technology, Daejeon 34114, Korea

${ }^{4}$ Department of Chemistry, University College London, 20 Gordon St., London WC1H OAJ, UK

+ These authors contributed equally to this work.

Corresponding Authors: sbhong@postech.ac.kr; xzou@mmk.su.se

\begin{abstract}
Zeolites have attracted great interest over recent decades. Their unique pore structures of molecular dimensions and tunable compositions make them ideal for shape selective catalysis and separation. However, targeted synthesis of zeolites with new pore structures and compositions remains a key challenge. Here, we propose a novel approach based on a unique 3D-3D topotactic transformation, which takes advantage of weak bonding in zeolites. This is inspired by the structure transformation of PST-5, a new aluminophosphate molecular sieve, to PST-6 by calcination at $500{ }^{\circ} \mathrm{C}$. The structure of PST- 5 was determined from micrometersized crystals by 3D electron diffraction (3DED, also known as MicroED). We found that the 3D-3D topotactic transformation involves two types of building units where penta- or hexacoordinated Al is present. We applied this approach to several other zeolite systems and predicted a series of new zeolite structures that would be synthetically feasible. This method provides a new concept for the synthesis of targeted zeolites, especially those which may not be feasible by conventional methods.
\end{abstract}




\section{Introduction}

Zeolites and related microporous materials have been widely used as selective catalysts, absorbents and ion-exchangers ${ }^{1-4}$. Recently zeolites have gained interest as catalysts for biomass conversion $^{5-8}$ and automotive exhaust remediation ${ }^{9,10}$. The unique properties of zeolites are associated with the well-defined pores and channels of molecular dimensions. Because of the close structure-property relationship, numerous efforts have been made to synthesize zeolitic materials with new pore structures and compositions and subsequently explore their properties and applications. Currently, only 252 zeolite framework topologies have been approved by the International Zeolite Association ${ }^{11}$, even though millions of possible zeolite topologies have been identified by computational methods ${ }^{12,13}$. The huge gap between experimental output and theoretical prediction indicates that our understanding of zeolite crystallization is still limited. It is therefore important to push the field by developing rational strategies to synthesize new zeolites. Besides the conventional hydro-/solvothermal synthesis, several new synthetic strategies have been proposed for targeted zeolite synthesis. These include bottom-up approaches by using e.g., pre-designed organic structure-directing agents $(\text { OSDA })^{14-17}$ and promotion of specific cavities as in the synthesis of embedded isoreticular zeolites, ${ }^{18,19}$ and top-down approaches, i.e., topotactic transformations. The most common topdown approach to synthesize new zeolites has been 2D-3D layer condensation from a layered precursor. ${ }^{20-23}$ Recently, a new approach of using known zeolites as precursors to synthesize new zeolites via 3D-2D-3D topotactic transformation has gained significant attention. ${ }^{24-27}$ The most successful example of these methods is the assembly-disassembly-organizationreassembly (ADOR) synthesis route. The unique concept of the ADOR process is to exploit chemically selective weak $\mathrm{T}-\mathrm{O}$ bonds ( $\mathrm{T}=\mathrm{Al}, \mathrm{Si}, \mathrm{Ge}$, etc.), e.g., Ge-O bonds in germanosilicates, in known zeolites and subsequently promote the formation of new T-O bonds. The ADOR strategy provides a synthetic tool for the generation of zeolites with novel topologies and compositions that cannot be synthesized by conventional hydrothermal methods. To the best of our knowledge, these targeted synthesis strategies have only been demonstrated on silica-based zeolite syntheses (e.g., silicates, aluminosilicates and germanosilicates), but never on phosphate-based frameworks.

Aluminophosphates (known as $\mathrm{AlPO}_{4}-n$ ), first synthesized by Union Carbide ${ }^{28}$, are an important class of zeolitic molecular sieves with wide applications. $\mathrm{AlPO}_{4}$ molecular sieves have a unique feature, which allows substitution of the Al or P by main block elements (e.g., $\mathrm{Si}$ ) or transition metals to engender specific properties in the material ${ }^{29,30}$. For example, silicoaluminophosphate SAPO-34 (framework type CHA) is one of the most widely used solid catalysts for biomass conversion, with a global market of tens of billions of US dollars per year. Currently, only $20 \%$ of the zeolite framework types can be synthesized as $\mathrm{AlPO}_{4}$ phases ${ }^{11}$, and examples of rational synthesis of $\mathrm{AlPO}_{4}$ materials are very rare. ${ }^{31}$ In $\mathrm{AlPO}_{4}$ frameworks, the $\mathrm{AlO}_{4}$ and $\mathrm{PO}_{4}$ tetrahedra always alternate according to Löwenstein's rule ${ }^{32}$. Nevertheless, $\mathrm{Al}$ 
can adopt penta-coordination with a pair of $\mathrm{Al}$ atoms bridged by a hydroxyl group as found in $\mathrm{AlPO}_{4}-21$ (AWO) ${ }^{33}$ and hexa-coordination with two additional water molecules, for example, in $\mathrm{AlPO}_{4}-\mathrm{C}(\mathrm{APC})^{34}$. Upon the loss of water molecules by heating, $\mathrm{AlPO}_{4}-21$ and $\mathrm{AlPO}_{4}-\mathrm{C}$ transform into new fully tetrahedrally-coordinated zeolite frameworks $\mathrm{AlPO}_{4}-25$ (ATV) and $\mathrm{AlPO}_{4}$-D (APD), respectively. In 2014, we reported the synthesis and structure of a new $\mathrm{AlPO}_{4}$ zeolite denoted PST-6. ${ }^{35}$ The framework of PST-6 (PSI) has a one-dimensional (1D) pore system containing parallel 8- and 10-ring channels. PST-6 was obtained by calcination of PST5 at $500{ }^{\circ} \mathrm{C}$, which was synthesized using diethylamine (DEA) as an OSDA.

Both PST-5 and PST-6 are obtained as nano-size plate-like crystals (Fig. S1). The structure of PST-6 was successfully solved by combining electron diffraction and powder X-ray diffraction (PXRD). However, the poorer stability of PST-5 under the electron beam prevented its structure determination, and therefore the understanding of its structure transformation to PST-6. Solid-state nuclear magnetic resonance (NMR) spectroscopy indicated the presence of bridging hydroxyl groups in PST-5.

Recent developments of fast 3D electron diffraction data collection techniques $(<5 \mathrm{~min})$ using continuous rotation (denoted $c$ RED here) have made it possible for studying beam sensitive nano- and micrometer-sized crystals ${ }^{18,36-40}$. Here we demonstrate the $a b$ initio structure determination of PST-5 using $c$ RED data collected under low dose condition. By detailed investigations of the structure transformation of PST-5 to PST-6, we propose a new approach to generate novel zeolite structures from known zeolites via 3D-3D topotactic transformation. The transformation was initiated via the weak Al-O bonding in pentacoordinated $\mathrm{AlO}_{4}(\mathrm{OH})$ pairs or hexa-coordinated $\mathrm{AlO}_{4}(\mathrm{OH})_{2}$ units. By adopting this approach, we have, in addition, predicted a series of targeted hypothetical zeolite structures.

\section{Results and discussion}

A high-resolution $c$ RED dataset of PST-5 (with a resolution better than $0.85 \AA$ ) was collected from a sub-micrometer sized crystal using low dose and fast data collection (in total 15 e $\AA^{-2}$ and 3 min) on a JEOL JEM2100 transmission electron microscope (Figs. 1a and S2). PST-5 crystallizes in an orthorhombic space group $\mathrm{Pb} 2 b$ (No. 27), with the unit cell parameters $a=36.5956(2) \AA, b=21.80273(9) \AA, c=10.26929$ (4) $\AA$ (Tables S1 and S2). Its framework structure was first solved $a b$ initio by direct methods. To confirm the model and obtain more accurate positions and atomic displace parameters (ADPs) of individual atoms, the model was further refined against the $c$ RED data (Table S2). Details of structure determination are given in the Supplementary Information. The framework of PST-5 exhibits a totally new topology with a fully four-connected 3D net and a 2D pore system containing parallel 8 - $(5.5 \times 2.6 \AA)$ and 10-ring $(5.8 \times 4.4 \AA)$ channels along the $c$-axis and another 8-ring $(3.3 \times 3.3 \AA)$ channel along the $\boldsymbol{b}$-axis (Fig. 2). PST-5 is one of the most complex zeolite structures, with $18 \mathrm{Al}, 18$ $\mathrm{P}$, and $76 \mathrm{O}$ atoms in the asymmetric unit. Four of the $\mathrm{O}$ atoms belong to hydroxyl groups and act as bridging atoms to form Al-OH-Al linkages. All $18 \mathrm{P}$ atoms and 10 out of the $18 \mathrm{Al}$ atoms 
are tetrahedrally coordinated with $\mathrm{O}$ atom to form $\mathrm{TO}_{4}$ units $(\mathrm{T}=\mathrm{Al}, \mathrm{P})$. The remaining $8 \mathrm{Al}$ atoms have trigonal bipyramidal-coordination and form four symmetry-independent $\mathrm{AlO}_{4}$ $\mathrm{OH}-\mathrm{AlO}_{4}$ pairs in the structure. Each $\mathrm{AlO}_{4}-\mathrm{OH}-\mathrm{AlO}_{4}$ pair becomes part of a 3- and a 5-ring (Fig. S8). Solid-state NMR spectroscopy confirmed the presence of Al-O-Al linkages (Figs. S3-S6), and a sharp IR band appearing at $3398 \mathrm{~cm}^{-1}$ indicates the presence of bridging $\mathrm{OH}$ groups (Fig. S7). The structure model obtained by Rietveld refinement against synchrotron powder XRD data agrees well with that obtained from $c$ RED data, which further confirms the framework structure of PST-5. In addition, the OSDA positions in the channels could be located from both $c$ RED and PXRD data, and subsequently refined by Rietveld refinement. The final refined unit cell composition is $\left.\mid\left(\mathrm{C}_{2} \mathrm{H}_{5}\right)_{2} \mathrm{NH}_{2}{ }^{+}\right)_{16} \mid\left[\mathrm{Al}_{72} \mathrm{P}_{72} \mathrm{O}_{288}\left(\mathrm{OH}^{-}\right)_{16}\right]$ (Figs. $1 \mathrm{~b}$ and S9, Table S3, see Supplementary Information for more details). Raman spectrum shows that DEA molecules are protonated during PST-5 crystallization ${ }^{35}$. Therefore, the negative framework charges created by the bridging Al-OH-Al groups are balanced by the protonated form of the occluded OSDA molecules $\left(\mathrm{DEA} \cdot \mathrm{H}^{+}\right)$, as reflected by quite similar numbers of OSDA molecules and bridging $\mathrm{O}$ atoms.
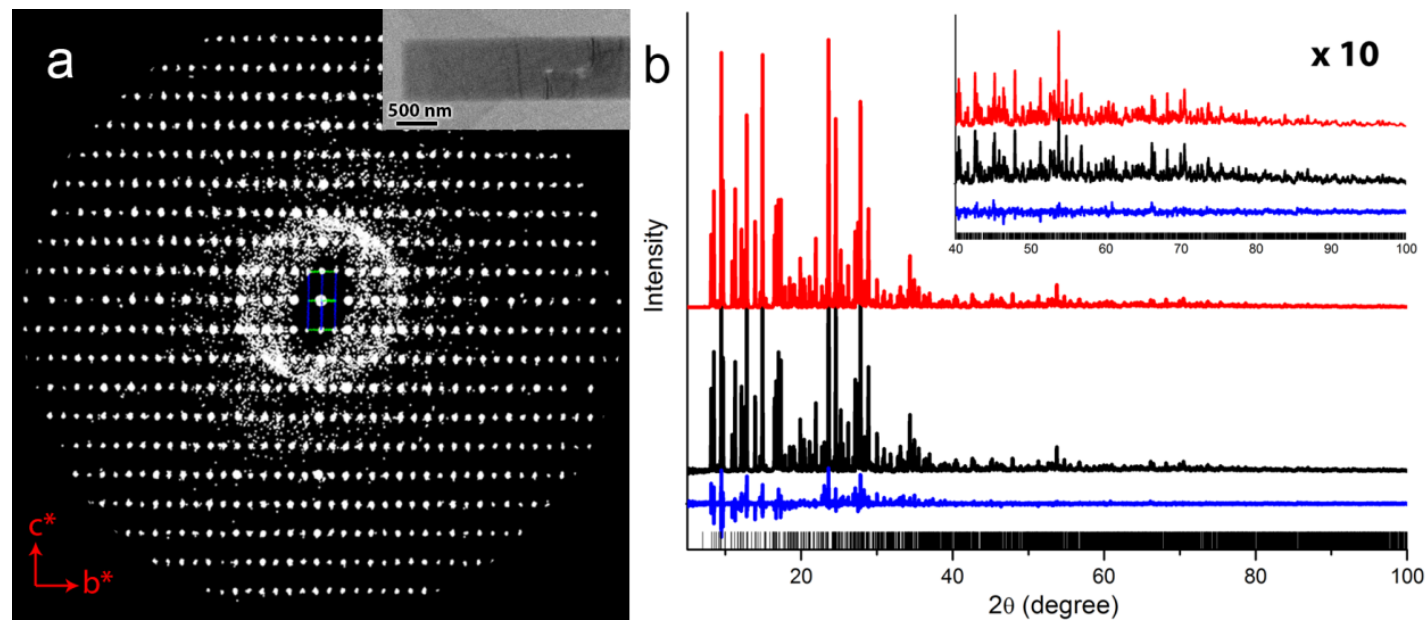

Figure 1 | Reconstructed 3D reciprocal lattice and Rietveld refinement. (a) Reconstructed 3D reciprocal lattice of PST-5 from the cRED data viewed along [100]. Inset is the crystal of PST-5 from which the cRED data was collected. (b) XRD profiles of Rietveld refinement for PST-5 ( $\lambda=1.5474 \AA$ ): observed, calculated and difference profiles are shown in red, black and blue, respectively. The bars represent the positions of allowed reflections.

In situ PXRD patterns indicate that the structural transformation of PST-5 to PST-6 starts at $200{ }^{\circ} \mathrm{C}$, and finishes at $500{ }^{\circ} \mathrm{C}$ (Fig. S10), which is accompanied by notable changes in the PXRD pattern. The observed pattern after calcination of PST-5 at $500^{\circ} \mathrm{C}$ agrees very well with the simulated pattern from the PST-6 model, confirming the calcined PST-5(i.e., PST-6) has the PSI framework (Fig. S11). In-situ IR studies show that dehydroxylation of bridging $\mathrm{OH}$ groups in PST-5 starts above $200{ }^{\circ} \mathrm{C}$ and finishes at $300{ }^{\circ} \mathrm{C}$ (Fig. S3, see SI for more details). Both structures are built from a similar building layer containing 4-, 6-, 8- and 10-rings if not considering the $\mathrm{OH}$ groups (Figs. $2 \mathrm{a}_{1}$ and $\mathrm{b}_{1}$ ). Interestingly, the transformation of PST-5 to 
PST-6 consists only in the loss not only with the loss of the hydroxyl groups, as observed in the transformation of PST-13 to PST- $14 .{ }^{41}$ Additionally, it involves T-O bond-breaking and reforming, which leads to totally different connections of the building layers in the two structures (Figs. 2a $\mathrm{a}_{2}$ and $\mathrm{b}_{2}$ ) and thereby different pore systems; 1D for PST-6, in contrast to 2D for PST-5.

The mechanism for the 3D-3D topotactic transformation of PST-5 to PST-6 is investigated based on their structure relationships as well as molecular dynamics simulations. PST-5 and PST-6 show large structure similarities when viewed along the $c$-axis (Figs. $2 \mathrm{a}_{1}$ and $\mathrm{b}_{1}$ ). The framework of PST-5 consists mainly of two types of building units; double-crankshaft chain $(d c c)$ and double 4-ring $(d 4 r)$, both contain 4-rings, as shown in Fig. 2c and highlighted in Fig. $2 \mathrm{a}_{1}$. The framework of PST-6 consists mainly of one type of building unit; a narsarsukite-type chain (nsc), as shown in Fig. $2 \mathrm{c}$ and marked in Fig. 2 $\mathrm{b}_{1}$. The transformation of PST-5 to PST6 can be described by the $d c c-n s c$ and $d 4 r$-nsc transformations. It is worth noting that the $d c c$ $n s c$ transformation was also observed in $\mathrm{AlPO}_{4}-21$ to $\mathrm{AlPO}_{4}-25^{33}$, and $\mathrm{AlPO}_{4}-\mathrm{C}$ to $\mathrm{AlPO}_{4}-\mathrm{D}^{34}$. However, the $d 4 r-n s c$ transformation has not been reported before.
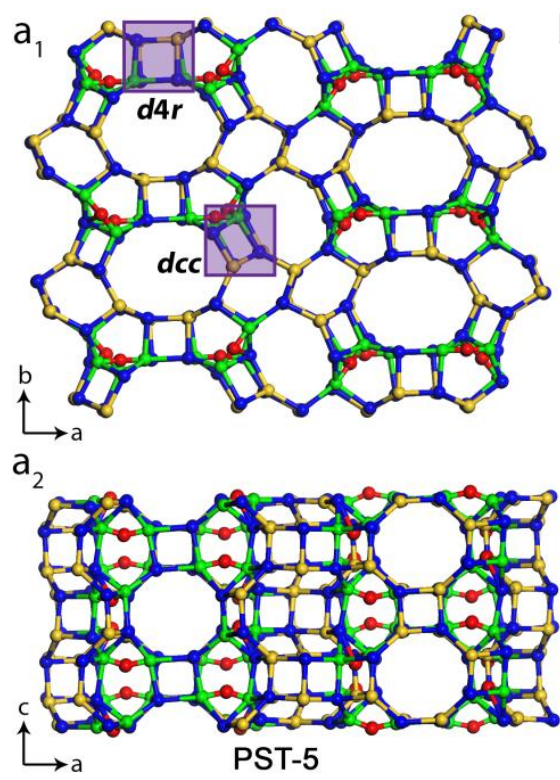

$\mathrm{b}_{1}$

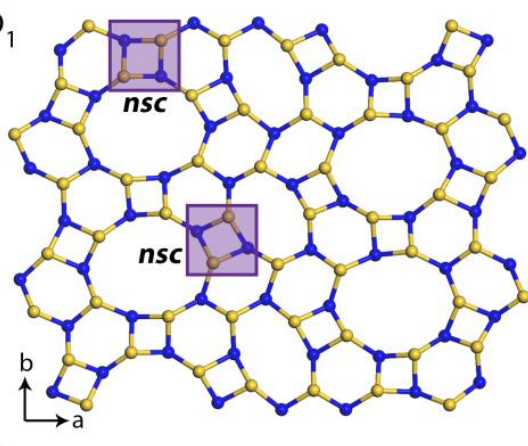

$b_{2}$

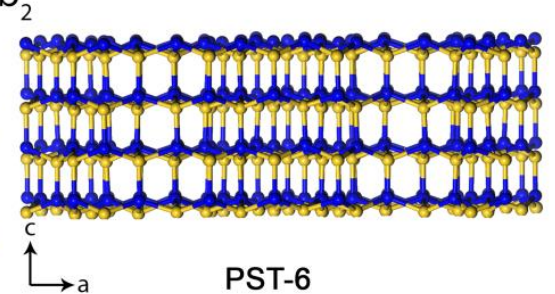

c

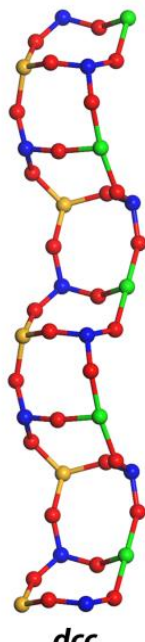

dcc

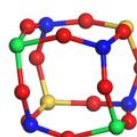

d4r
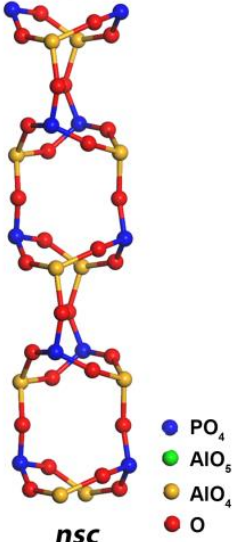

Figure 2 | Comparison of the structures of PST-5 and PST-6. Structural models of PST-5 $\left(a_{1}\right)$ and PST$6\left(b_{1}\right)$ viewed along the $c$-axis. Both structures are built from the same building layer containing 4-, 6-, 8- and 10-rings (if the $\mathrm{OH}$ groups, shown in red are not considered). The difference is the orientations of the $(\mathrm{Al}, \mathrm{P}) \mathrm{O}_{4}$ tetrahedra in the two structures. The red atoms in $\left(\mathrm{a}_{1}\right)$ correspond to the $\mathrm{OH}$ groups bridging two Al atoms in PST-5. These Al atoms are five-coordinated. Structural models of PST-5 $\left(a_{2}\right)$ and PST-6 $\left(\mathrm{b}_{2}\right)$ viewed along the $b$-axis, showing the transforms of double-crankshaft chains $(d c c)$ and double 4-rings ( $d 4 r)$ in PST-5 $\left(\mathrm{a}_{1}\right)$ to narsarsukite-type chains ( $\left.n s c\right)$ in PST-6 (indicated by purple boxes). (c) Building units in PST-5 (dcc and $d 4 r$ ) and PST-6 (nsc).

The key to the chain transformation is the penta-coordinated $\mathrm{Al}$ atoms, where $\mathrm{Al}-\mathrm{O}$ bond lengths (on average 1.83(9) $\AA$ ) are longer than those of tetra-coordinated Al atoms (on average 1.72(1) Å). The longer bond lengths indicate weaker bonds, which are more easily broken. 
Therefore, the built-in weakness associated with penta-coordinated $\mathrm{Al}$ atoms in the structure may be utilized to initiate the topotactic transformation. Upon thermal treatment, the bridging hydroxyl groups are removed through a dehydroxylation to form the fully four-connected framework PST-6 (Figs. 2 and S8).

By detailed inspection of the structural relationships between PST-5 and PST-6, the geometry changes of penta-coordinated $\mathrm{Al}$ atoms initiate a domino geometrical inversion of nearby $\mathrm{PO}_{4}$ tetrahedra. Upon heating, the penta-coordinated $\mathrm{Al}$ atoms lose the bridging hydroxyl groups, which also leads to cleavage of the Al-O-P bond to the adjacent $\mathrm{PO}_{4}$ tetrahedra. The $\mathrm{AlO}_{4}$ and $\mathrm{PO}_{4}$ tetrahedral pairs with resulting broken bonds then invert their orientations and reconnect to the next nearest pairs of $\mathrm{AlO}_{4}$ and $\mathrm{PO}_{4}$ tetrahedra (Fig. 3, indicated by arrows). As a result, the double-crankshaft chains (dcc) in PST-5 transforms to the narsarsukite-type chains (nsc) in PST-6, which is composed of one chain with tetracoordinated $\mathrm{Al}$ atoms, and another chain originating from penta-coordinated $\mathrm{Al}$ atoms (Figs. $3 a_{1}$ and $b_{1}$ ). The inversion further extends to neighboring atoms, which results in a domino-like alteration of the tetrahedral conformation. There are two symmetry-independent $d c c$ chains in PST-5 with different orientations. After being transformed to PST-6, they orient in the same way. The reconstructed PST-6 (PSI) material was found to contain stacking faults ${ }^{35}$, due to changes in both orientation and tetrahedral conformation. There are eight unique pentacoordinated $\mathrm{Al}$ atoms that can initiate the topotactic transformation. When the transformation starts at different positions, it will also generate disorder after the reconstruction.
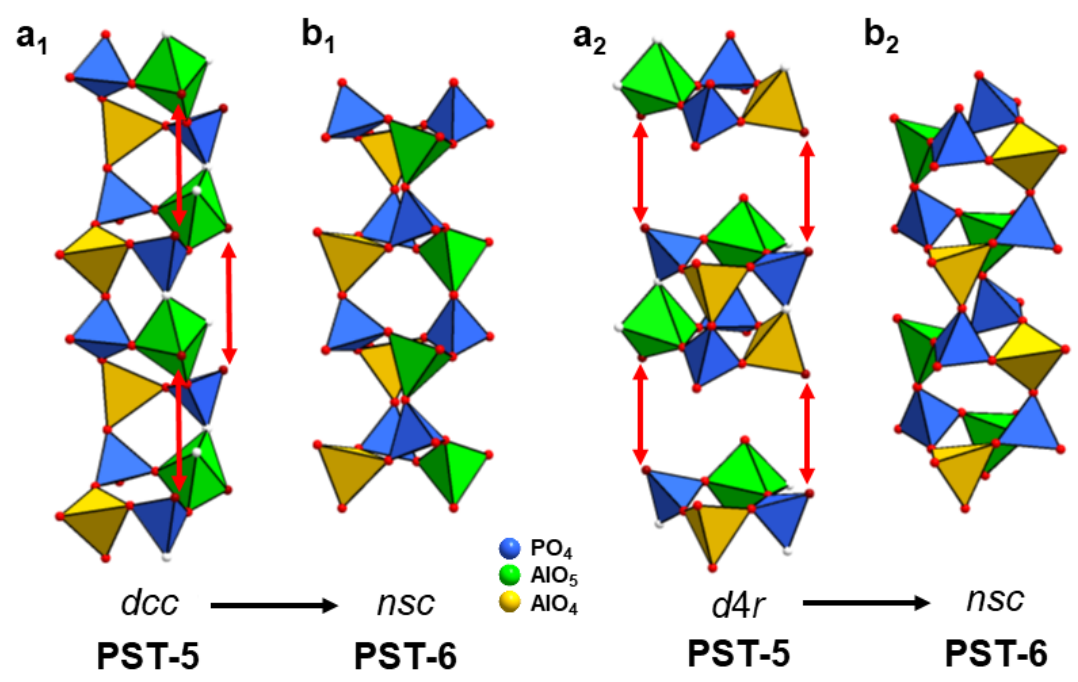

Figure 3 | Chain transformations in the topotactic transformation between PST-5 and PST6. $\left(\mathrm{a}_{1}\right) d c c$ and $\left(\mathrm{a}_{2}\right) d 4 r$ unit in PST-5, and ( $\mathrm{b}_{1}$ and $\left.\mathrm{b}_{2}\right) n s c$ in PST-6. The removal of bridging hydroxyl groups associated with the $d c c$ and $d 4 r$ initiates the geometry changes of pentacoordinated $\mathrm{Al}$ atoms, resulting in cleavage of $\mathrm{Al}-\mathrm{O}-\mathrm{P}$ bonds (corresponding $\mathrm{O}$ atoms are shown in white) followed by inversion and reconnection of the tetrahedra (indicated by arrows). Both $d c c$ and $d 4 r$ transform to $n s c$ in PST-6. Green, penta-coordinated (and penta-coordinated derived) $\mathrm{AlO}_{4}$ tetrahedra; yellow, tetra-coordinated $\mathrm{AlO}_{4}$; blue, $\mathrm{PO}_{4}$. Oxygen atoms associated with $\mathrm{OH}$ or bond breaking in the chains are shown in white and the rest 0 in red. 
To study the possible mechanisms for the transformation of PST-5 to PST-6, periodic $a b$ initio molecular dynamics simulations were carried out on a model PST-5 system. In nearly every case, one bond from penta-coordinated $\mathrm{Al}$ was broken, forming tetra-coordinated $\mathrm{Al}$ and corresponding P-OH groups (Figs. S12 and S13). In one case, the Al-O-P linkage was broken within a 3- (-Al-O-P-Al-OH-) ring, but otherwise the framework interruption occurred between the penta-coordinated $\mathrm{Al}$ and neighboring $\mathrm{P}$ atoms, which were part of a double-crankshaft chain $(d c c)$ or a double-4-ring $(d 4 r)$ structural unit but not in a 3-ring. The simulations clearly confirm that weaker bonds in the PST-5 framework are associated with penta-coordinated Al atoms and are labile in the presence of an extra-framework proton donor. The initial broken bonds are those in the $d c c$ and $d 4 r$, largely the same as those implicated in the transformation of PST-5 to PST-6 at the experimental annealing temperature. Coupled with the topological strain inherent within the $d c c$ and $d 4 r$ due to their fused 4-ring motifs, the penta-coordinated $\mathrm{Al}$ atoms are thus critical in introducing reactivity into the framework. It should be noted that the molecular dynamics simulations of an idealised, fully four-connected, $\mathrm{AlPO}_{4} \mathrm{PST}-5$ framework, without penta-coordinated $\mathrm{Al}$ atoms and bridging $\mathrm{OH}$ groups, showed no cleavage of any framework bonds over the same timescale at $500{ }^{\circ} \mathrm{C}$.

Inspired by the 3D-3D topotatic transformation in PST-5/PST-6, we investigated the existing structures in the Zeolite Structure Database ${ }^{11} .12$ framework types are found to contain infinite $d c c$, and they can be divided into three groups based on the structural complexity (Table 1): I) built solely from $d c c$ that are connected directly (APC, GIS, MER, PHI, SIV, GME, Fig. S14); II) built from $d c c$ that are bridged by isolated 4-rings (ATT, AWO, UEI, Figs. S15 and S17); III) built by $d c c$ and other building units (PST-5, DON, STO, Fig. S16). Interestingly, regardless of the complexity, the $d c c$-nsc structural transformation has been observed in all groups, from $\mathrm{AlPO}_{4}-\mathrm{C}(\mathrm{APC})$ to $\mathrm{AlPO}_{4}-\mathrm{D}$ (APD) in group I, AlPO 4 -21 (AWO) to $\mathrm{AlPO}_{4}-25$ (ATV) in group II, and PST-5 to PST-6 (PSI) in group III (Tables 1 and S4). The three parent frameworks all contain $d c c$ in which penta- $\left(\mathrm{AlPO}_{4}-21\right.$ and PST-5) or hexa- $\left(\mathrm{AlPO}_{4}-\mathrm{C}\right)$ coordinated $\mathrm{Al}$ atoms are present. By applying similar $d c c$ to $n s c$ transformations observed in the three known zeolite pairs to other $d c c$-containing zeolites, we generated a series of novel hypothetical $n s c$-containing zeolite structures, as shown in Figs. 4, S14-18, Tables 1 and S5 (see Supplementary Information for more details). ${ }^{42}$ Notably, the same parent zeolite can generate more than one hypothetical zeolites (e.g. UEI to UEI_H1-5), and the same zeolite can be generated from different parent zeolites (e.g. GIS_HI from both GIS and SIV). The transformation can also generate new channel dimensionality (Fig. S18 and Table 1). All these hypothetical $n s c$-containing zeolites fulfill the criteria for zeolite frameworks realizable for target synthesis (Table S6). ${ }^{43}$ Most of them were calculated to have much lower framework energies than the parent $d c c$-containing zeolites. It is worth mentioning that because each $\mathrm{TO}_{4}$ tetrahedral unit not belonging to $d c c$ can have two possible connectivities, either upwards or downwards, the number of hypothetical zeolites generated via this approach can be enormous for zeolites in group II and III (see Supplementary Information for more details). 

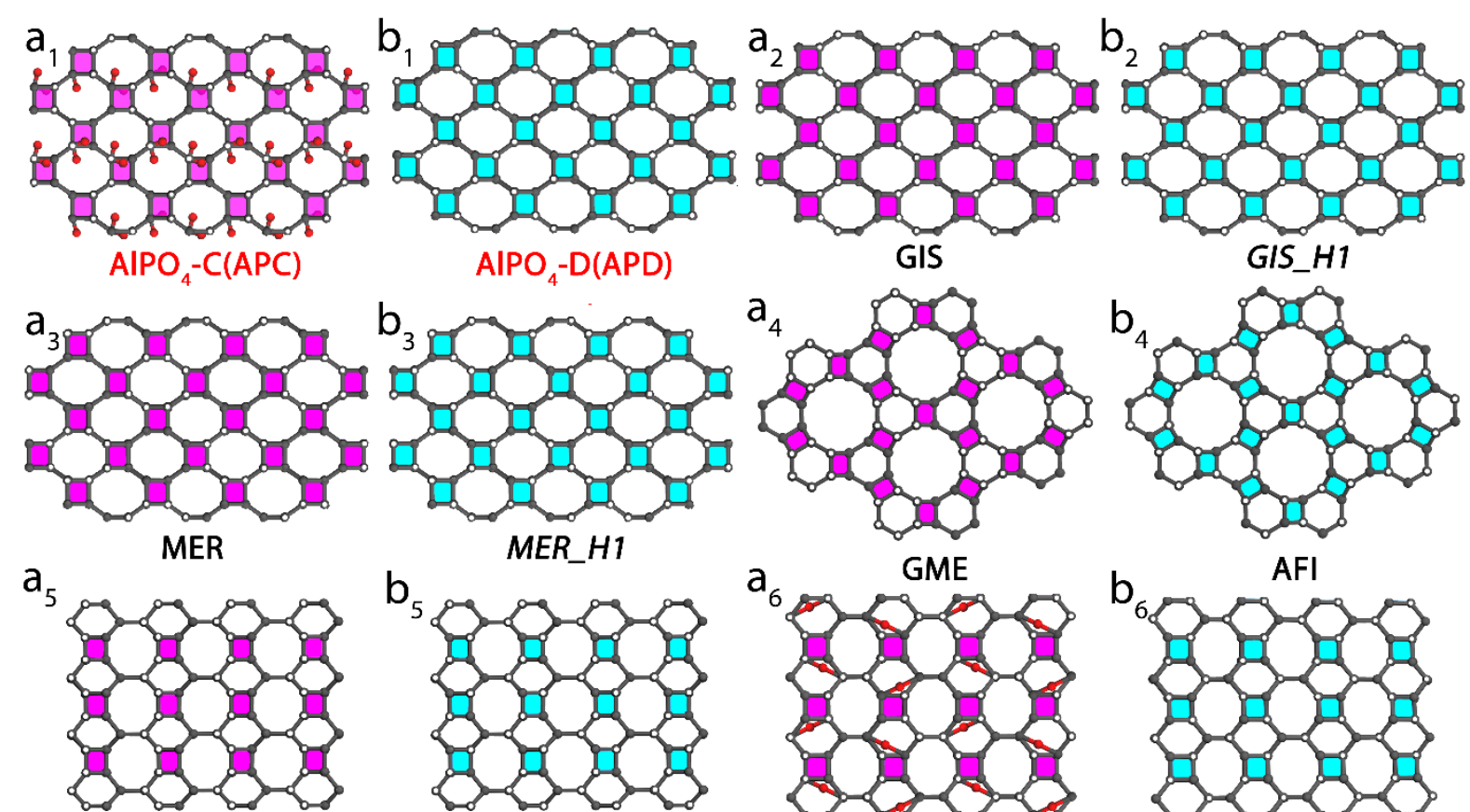

MER_H1
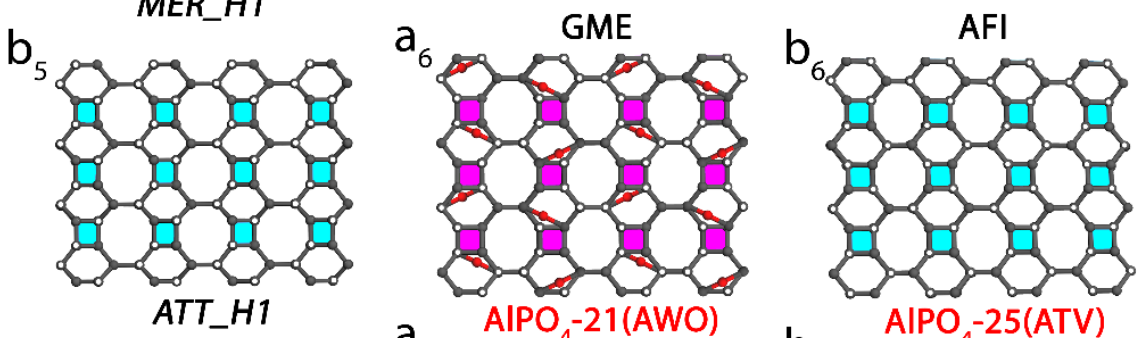

$a_{7}$

$b_{7}$
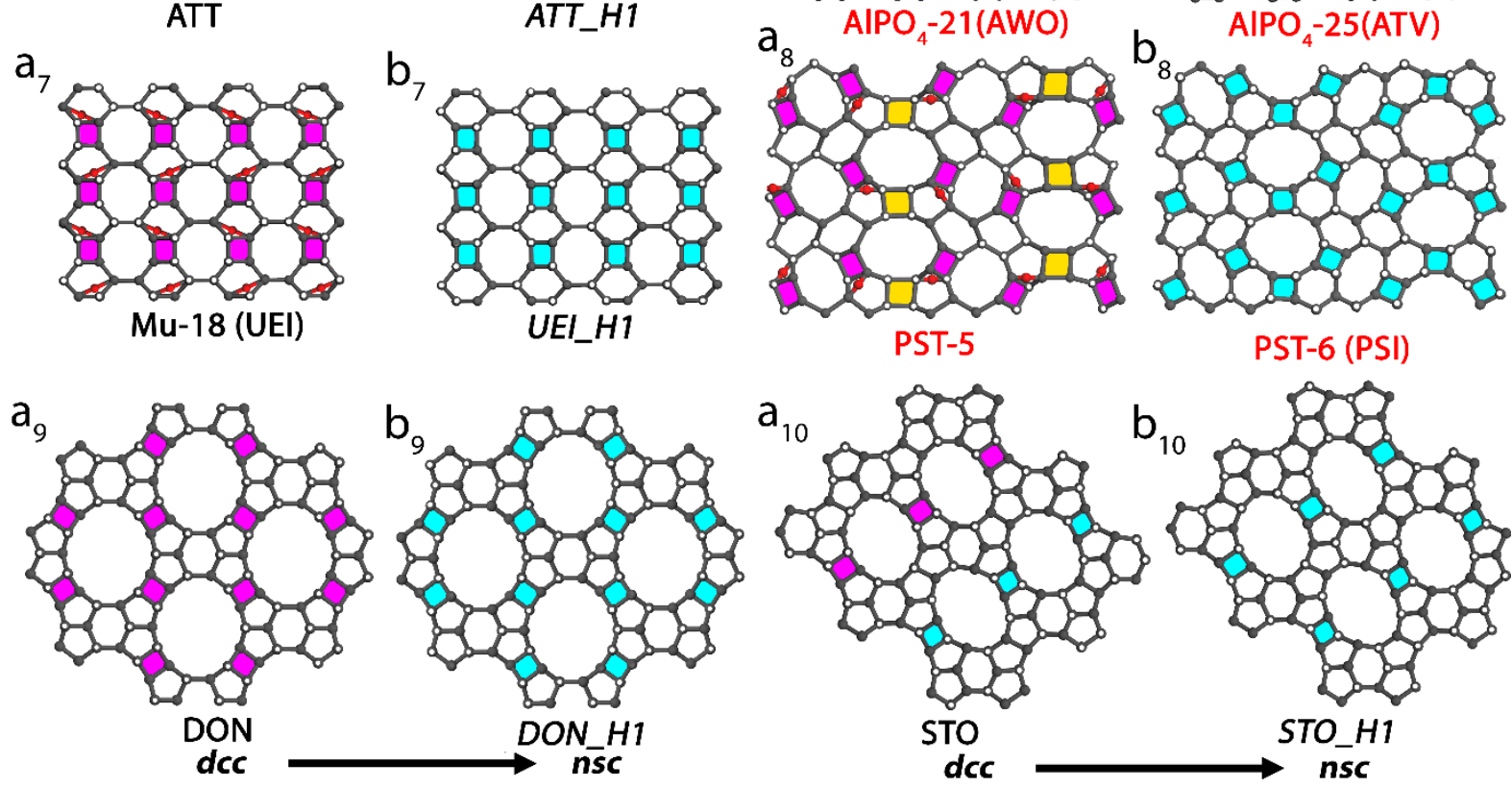

Figure 4 | Illustration of observed and predicted 3D-3D topotactic transformations from double-crankshaft chain $(\boldsymbol{d c c})$ to narsarsukite chain $(\boldsymbol{n s c}) .\left(\mathrm{a}_{1}-\mathrm{a}_{10}\right)$ Projections of the APC, GIS, MER, GME, ATT, AWO, UEI, PST-5, DON and STO frameworks. All of them contain dcc. Among them, APC, AWO, UEI, and PST-5 contain penta- or hexa-coordinated atoms. $\left(b_{1}, b_{6}\right.$ and $\left.b_{8}\right)$ Projections of APD, ATV and PSI (PST-6) frameworks after topotactic transformation by changing dcc to nsc. They have the same framework projections as APC, AWO and PST-5, respectively, but differ in the 3D connectivity along the projections. $\left(\mathrm{b}_{4}\right)\left(\mathrm{b}_{2}-\mathrm{b}_{5}, \mathrm{~b}_{7}, \mathrm{~b}_{9}\right.$ and $\left.\mathrm{b}_{10}\right)$ Selected hypothetical structures of GIS_H1, MER_H1, ATT_H1,UEI_H1, and DON_H1 generated by replacing dcc with nsc. Magenta: $d c c$; cyan: $n s c$; yellow: $d 4 r$. The solid and hollow circles indicate vertices that connect upwards and downwards, respectively. 
Table 1. Observed (in bold) and predicted 3D-3D topotactic transformations of zeolites through chain transformation from $d c c$ to $n s c$ and $d 4 r$ to $n s c$.

\begin{tabular}{|c|c|c|c|c|c|}
\hline FTC $^{[\mathrm{a}]}$ (parent) & $\mathrm{E}_{\mathrm{F}}^{[\mathrm{b}]}$ & $\begin{array}{c}\text { Channel } \\
\text { dimensionality }\end{array}$ & FTC (transformed) & $\mathrm{E}_{\mathrm{F}}{ }^{[\mathrm{b}]}$ & $\begin{array}{c}\text { Channel } \\
\text { dimensionality }\end{array}$ \\
\hline Group II: & $d c c$ & \multicolumn{2}{|c|}{$\rightarrow$} & \multicolumn{2}{|l|}{$n s c$} \\
\hline $\mathrm{APC}$ & 14.4 & 2D & APD & 12.3 & $2 D$ \\
\hline GIS & 15.0 & $3 \mathrm{D}$ & GIS_H1 & 10.3 & $1 \mathrm{D}$ \\
\hline SIV & 15.3 & $3 \mathrm{D}$ & GIS_HI & 10.3 & $1 \mathrm{D}$ \\
\hline MER & 16.2 & $3 \mathrm{D}$ & $M E R \_H 1$ & 12.3 & $2 \mathrm{D}$ \\
\hline PHI & 15.7 & $3 \mathrm{D}$ & $M E R \_H 1$ & 12.3 & $2 \mathrm{D}$ \\
\hline GME & 16.5 & $3 \mathrm{D}$ & AFI & 11.0 & $1 \mathrm{D}$ \\
\hline Group II: & \multicolumn{2}{|c|}{$d c c+$ isolated 4-rings } & & \multicolumn{2}{|c|}{$n s c+s c c^{[\mathrm{c}]}$} \\
\hline ATT & 15.1 & $2 \mathrm{D}$ & $A T T \_H 1$ & 10.8 & $2 \mathrm{D}$ \\
\hline AWO & 14.0 & $2 \mathrm{D}$ & $\begin{array}{l}\mathbf{A T V} \\
A W O \_H 1 \\
A W O \_H 2\end{array}$ & $\begin{array}{c}\mathbf{8 . 9} \\
12.2 \\
14.7\end{array}$ & $\begin{array}{l}1 D \\
1 D \\
1 D\end{array}$ \\
\hline UEI & 14.2 & $2 \mathrm{D}$ & $\begin{array}{l}\text { UEI_H1 } \\
U E I \_H 2 \\
U E I \_H 3 \\
U E I \_H 4 \\
U E I \_H 5\end{array}$ & $\begin{array}{l}11.2 \\
11.3 \\
11.0 \\
11.3 \\
11.5\end{array}$ & $\begin{array}{l}1 \mathrm{D} \\
1 \mathrm{D} \\
1 \mathrm{D} \\
1 \mathrm{D} \\
1 \mathrm{D}\end{array}$ \\
\hline \multicolumn{3}{|c|}{ Group III: $d c c$ + other building units } & $n s c$ & \multicolumn{2}{|c|}{$n s c+$ other building units } \\
\hline PST-5 & 15.9 & $2 \mathrm{D}$ & PSI & 9.2 & $1 D$ \\
\hline DON & 10.2 & $1 \mathrm{D}$ & DON_HI & 13.2 & $1 \mathrm{D}$ \\
\hline STO & 9.7 & $1 \mathrm{D}$ & STO_H1 & 10.6 & $1 \mathrm{D}$ \\
\hline
\end{tabular}

[a] Framework Type Code approved by International Zeolite Association.

[b] Framework energy ( $\mathrm{kJ}$ (mol Si)-1) relative to $\alpha$-quartz.

[c] single crankshaft chain.

We have demonstrated that many hypothetical zeolites can be predicted from known zeolite frameworks based on the $d c c-n s c$ transformation. The 3D-3D topotactic transformation would provide a simple approach to generate novel zeolite frameworks, which may be inaccessible by conventional hydrothermal/solvothermal synthesis. We have also shown by molecular dynamics simulations that the weakened bonding, such as penta- or hexacoordinated $\mathrm{Al}$ atoms, is crucial to initiate the 3D-3D topotactic transformation. Therefore, to synthesize new structures by this approach, it is important to introduce penta- or hexacoordinated $\mathrm{Al}$ atoms in $d c c$-containing zeolite frameworks, and additional exploratory work is needed. Previous studies showed that certain types of OSDAs, like easily protonatable amines, could promote the formation of bridging $\mathrm{Al}-\mathrm{OH}-\mathrm{Al}$ bonds in $\mathrm{AlPO}_{4}$ molecular sieves, and penta- or hexa-coordinated $\mathrm{Al}$ atoms have been found in c.a. $25 \%$ of these materials, including $\mathrm{AlPO}_{4}-17, \mathrm{AlPO}_{4}-41, \mathrm{AlPO}_{4}-\mathrm{EN} 3, \mathrm{AlPO}-\mathrm{CJB} 1, \mathrm{VPI}-5$, IST-1, PST-13, etc. ${ }^{41,44-50}$ The 3D-3D topotactic transformation may also be applicable to other zeolite compositions. For example, the gallophosphate Mu-18 (UEI) contains both $d c c$ and penta-coordinated atoms (Fig. 
$\left.4 a_{6}\right)^{51}$. We anticipate that with the discovery of many new zeolites, the approach described here will be more feasible.

\section{Conclusions}

We have solved the structure of PST-5, a new 2D medium-pore $\mathrm{AlPO}_{4}$ molecular sieve, using continuous rotation electron diffraction ( $c$ RED). Based on the investigations of the $d c c$ to $n s c$ transformations observed in PST-5/PST-6, AlPO 4 -C/AlPO 4 -D, and $\mathrm{AlPO}_{4}-21 / \mathrm{AlPO}_{4}-25$ pairs, we propose a new approach to generate novel zeolite structures, which enriches the structural diversity. The 3D-3D topotactic transformation relies on the chemical weakness in the $d c c$. PST- 5 transforms $d c c$ and $d 4 r$ to $n s c$, changes its overall interlayer connectivities and finally generates PST-6. The penta-coordinated Al atoms, as observed in PST-5, could be built into a precursor material by using pre-designed OSDAs which balance the framework energy. This new approach via the 3D-3D topotactic transformation can be considered complementary to the existing strategies of synthesizing novel zeolite structures. By understanding the 3D-3D topotactic transformation, we anticipate that our approach be generalized and provide new insights into the discovery of novel zeolitic materials, which may enable a completely new range of zeolite frameworks to be accessible and offer new opportunities in technological applications.

\section{Methods}

Synthesis of PST-5. PST-5 was synthesized from an $\mathrm{AlPO}_{4}$ gel with the composition of 2.0DEA $\cdot 1.0 \mathrm{Al}_{2} \mathrm{O}_{3} \cdot 1.0 \mathrm{P}_{2} \mathrm{O}_{5} \cdot 40 \mathrm{H}_{2} \mathrm{O}$, where DEA is diethylamine. In a typical synthesis of PST$5,3.84 \mathrm{~g}$ of $o-\mathrm{H}_{3} \mathrm{PO}_{4}\left(85 \%\right.$, Merck) was diluted in $5.63 \mathrm{~g}$ of $\mathrm{H}_{2} \mathrm{O}$. This solution was added dropwise to a slurry of $6.95 \mathrm{~g}$ of aluminum isopropoxide ( $>98 \%$, Aldrich) in $5.64 \mathrm{~g}$ of $\mathrm{H}_{2} \mathrm{O}$. After $1 \mathrm{~h}$ stirring, $2.45 \mathrm{~g}$ of DEA (99.5\%, Aldrich) was added to the above synthesis mixture, which was stirred overnight at room temperature. Afterwards, the mixture was charged into a Teflon-lined $23-\mathrm{mL}$ autoclave and heated under static conditions at $200{ }^{\circ} \mathrm{C}$ for 5 days. The resulting white powder was recovered by filtration, washed repeatedly with water and then dried overnight at room temperature.

Topotactic Transformation of PST-5. The topotactic transformation of PST-5 to PST-6 was conducted by calcination of PST- 5 at $550{ }^{\circ} \mathrm{C}$ for $8 \mathrm{~h}$ under air flow.

$\boldsymbol{c}$ RED Data Collection. PST-5 powder in its as-made form was dispersed in ethanol and a droplet of the suspension was transferred onto a carbon-coated copper grid and dried in air. $c$ RED data was acquired in selected-area mode with a goniometer tilt speed of $0.45^{\circ} \mathrm{s}^{-1}$ and an exposure time of $0.4 \mathrm{~s}$ per frame (Table S2). The total data collection time was less than 3 min in order to minimize the beam damage and maximize the data quality. The total tilt range was $92.6^{\circ}$.

\section{Acknowledgments}

This work was supported by the Swedish Research Council (VR, 2017-04321, 2016-04625), the Knut and Alice Wallenberg Foundation (KAW, 2012-0112) through the 3DEM-NATUR project and National Creative Research Initiative Program (2012R1A3A-2048833) through the 
National Research Foundation of Korea and the National Research Council of Science \& Technology (CRC-14-1-KRICT) grant by the Korea government (MSIP). We thank PAL (Pohang, Korea) for synchrotron diffraction beam time at beamline 9B (D. Ahn). PAL is supported by MSIP and POSTECH. We also thank G. J. Kennedy (ExxonMobil) for the ${ }^{27} \mathrm{Al}$ 3Q MAS and ${ }^{27} \mathrm{Al} \rightarrow{ }^{31} \mathrm{P}$ 3Q HETCOR NMR measurements.

\section{Author contributions}

Original idea was conceived by X.Z., S.B.H., Z.H. and S.S.; synthesis work was performed by S.S. and J.S., structural characterization was performed by Z.H. and B.W.; Rietveld refinement was performed by S.S. and J.S.; data analysis was performed by Z.H., S.S. and J.S.; molecular simulations were performed by R.B.; new structure generation was performed by Z.H. and S.S.; manuscript was drafted by Z.H., S.S., X.Z., S.B.H., R.B., J.S. and B.W. All authors have given approval to the manuscript.

\section{Data Availability}

The crystallographic data for PST-5 have been deposited at the Cambridge Crystallographic Data Centre (CCDC, free for charge at https://www.ccdc.cam.ac.uk) under deposition number CCDC 1948775 and 1944918. Supplementary information is available in the online version of the paper. Reprints and permissions information is available online at www.nature.com/reprints. Correspondence and requests for materials should be addressed to S.B.H. (sbhong@postech.ac.kr) and X.Z. (xzou@mmk.su.se).

\section{Competing financial interests}

The authors declare no competing financial interests.

\section{Reference}

1. Climent, M. J., Corma, A. \& Iborra, S. Heterogeneous Catalysts for the One-Pot Synthesis of Chemicals and Fine Chemicals. Chem. Rev. 111, 1072-1133 (2011).

2. Chen, L.-H. et al. Hierarchically structured zeolites: synthesis, mass transport properties and applications. J. Mater. Chem. 22, 17381-17403 (2012).

3. Dusselier, M. \& Davis, M. E. Small-Pore Zeolites: Synthesis and Catalysis. Chem. Rev. 118, 5265-5329 (2018).

4. Datta, S. J. et al. $\mathrm{CO} 2$ capture from humid flue gases and humid atmosphere using a microporous coppersilicate. Science 350, 302-306 (2015).

5. Yarulina, I., Chowdhury, A. D., Meirer, F., Weckhuysen, B. M. \& Gascon, J. Recent trends and fundamental insights in the methanol-to-hydrocarbons process. Nat. Catal. 1, 398 (2018).

6. Li, Y., Li, L. \& Yu, J. Applications of Zeolites in Sustainable Chemistry. Chem 3, 928-949 (2017).

7. Lee, J. H. et al. Synthesis and Characterization of ERI-Type UZM-12 Zeolites and Their Methanol-to-Olefin Performance. J. Am. Chem. Soc. 132, 12971-12982 (2010).

8. Sudarsanam, P., Peeters, E., Makshina, E. V., Parvulescu, V. I. \& Sels, B. F. Advances in porous and nanoscale catalysts for viable biomass conversion. Chem. Soc. Rev. 48, 2366-2421 (2019).

9. Marberger, A. et al. Time-resolved copper speciation during selective catalytic reduction of NO on Cu-SSZ-13. Nat. Catal. 1, 221 (2018).

10. Zhang, L. et al. Recent advances in the preparation of zeolites for the selective catalytic reduction of NOx in diesel engines. React. Chem. Eng. 4, 975-985 (2019).

11. Baerlocher, C. \& McCusker, L. B. Database of Zeolite Structures. http://www.izastructure.org/databases/. 
12. Akporiaye, D. E. \& Price, G. D. Systematic enumeration of zeolite frameworks. Zeolites 9, 23-32 (1989).

13. Pophale, R., Cheeseman, P. A. \& Deem, M. W. A database of new zeolite-like materials. Phys. Chem. Chem. Phys. 13, 12407-12412 (2011).

14. Moliner, M., Rey, F. \& Corma, A. Towards the Rational Design of Efficient Organic StructureDirecting Agents for Zeolite Synthesis. Angew. Chem. Int. Ed. 52, 13880-13889 (2013).

15. Gallego, E. M. et al. "Ab initio" synthesis of zeolites for preestablished catalytic reactions. Science 355, 1051-1054 (2017).

16. Li, Y., Li, X., Liu, J., Duan, F. \& Yu, J. In silico prediction and screening of modular crystal structures via a high-throughput genomic approach. Nat. Commun. 6, ncomms9328 (2015).

17. Park, M. B., Cho, S. J. \& Hong, S. B. Synthesis of Aluminosilicate and Gallosilicate Zeolites via a Charge Density Mismatch Approach and Their Characterization. J. Am. Chem. Soc. 133, 19171934 (2011).

18. Guo, P. et al. A zeolite family with expanding structural complexity and embedded isoreticular structures. Nature 524, 74-78 (2015).

19. Shin, J. et al. Targeted Synthesis of Two Super-Complex Zeolites with Embedded Isoreticular Structures. Angew. Chem. Int. Ed. 55, 4928-4932 (2016).

20. Leonowicz, M. E., Lawton, J. A., Lawton, S. L. \& Rubin, M. K. MCM-22: A Molecular Sieve with Two Independent Multidimensional Channel Systems. Science 264, 1910-1913 (1994).

21. Zanardi, S. et al. Crystal Structure Determination of Zeolite Nu-6(2) and Its Layered Precursor Nu-6(1). Angew. Chem. Int. Ed. 43, 4933-4937 (2004).

22. Ikeda, T., Akiyama, Y., Oumi, Y., Kawai, A. \& Mizukami, F. The Topotactic Conversion of a Novel Layered Silicate into a New Framework Zeolite. Angew. Chem. Int. Ed. 43, 4892-4896 (2004).

23. Wang, Y. X., Gies, H., Marler, B. \& Müller, U. Synthesis and Crystal Structure of Zeolite RUB41 Obtained as Calcination Product of a Layered Precursor: a Systematic Approach to a New Synthesis Route. Chem. Mater. 17, 43-49 (2005).

24. Roth, W. J. et al. A family of zeolites with controlled pore size prepared using a top-down method. Nat. Chem. 5, 628-633 (2013).

25. Mazur, M. et al. Synthesis of 'unfeasible' zeolites. Nat. Chem. 8, 58-62 (2016).

26. Verheyen, E. et al. Design of zeolite by inverse sigma transformation. Nat. Mater. 11, 1059-1064 (2012).

27. Marler, B. \& Gies, H. Hydrous layer silicates as precursors for zeolites obtained through topotactic condensation: a review. Eur. J. Mineral. 24, 405-428 (2012).

28. Wilson, S. T., Lok, B. M., Messina, C. A., Cannan, T. R. \& Flanigen, E. M. Aluminophosphate molecular sieves: a new class of microporous crystalline inorganic solids. J. Am. Chem. Soc. 104, 1146-1147 (1982).

29. Yu, J. \& Xu, R. Insight into the construction of open-framework aluminophosphates. Chem. Soc. Rev. 35, 593-604 (2006).

30. Cheetham, A. K., Férey, G. \& Loiseau, T. Open-Framework Inorganic Materials. Angew. Chem. Int. Ed. 38, 3268-3292 (1999).

31. Broach, R. W. et al. New ABC-6 net molecular sieves ZnAPO-57 and ZnAPO-59: Framework charge density-induced transition from two- to three-dimensional porosity. Microporous Mesoporous Mater. 189, 49-63 (2014).

32. Loewenstein, $\mathrm{W}$. The distribution of aluminum in the tetrahedra of silicates and aluminates. $\mathrm{Am}$ Miner. 39, 92-96 (1954).

33. Richardson, J. W., Smith, J. V. \& Pluth, J. J. AlPO4-25: framework topology, topotactic transformation from AlPO4-21, and high-low displacive transition. J. Phys. Chem. 94, 3365 3367 (1990).

34. Keller, E. B., Meier, W. M. \& Kirchner, R. M. Synthesis, structures of AlPO4-C and AlPO4-D, and their topotactic transformation. Solid State Ion. 43, 93-102 (1990).

35. Lee, J. K. et al. An Aluminophosphate Molecular Sieve with 36 Crystallographically Distinct Tetrahedral Sites. Angew. Chem. Int. Ed. 53, 7480-7483 (2014). 
36. Warren, M. 'Why didn't we think to do this earlier?' Chemists thrilled by speedy atomic structures. Nature 563, 16 (2018).

37. Brázda, P., Palatinus, L. \& Babor, M. Electron diffraction determines molecular absolute configuration in a pharmaceutical nanocrystal. Science 364, 667-669 (2019).

38. Sun, J. et al. The ITQ-37 mesoporous chiral zeolite. Nature 458, 1154-1157 (2009).

39. Yuan, S. et al. [Ti8Zr2O12(COO)16] Cluster: An Ideal Inorganic Building Unit for Photoactive Metal-Organic Frameworks. ACS Cent. Sci. 4, 105-111 (2018).

40. Huang, Z. et al. Can 3D Electron Diffraction Provide Accurate Atomic Structures of MetalOrganic Frameworks? Faraday Discuss. (2020) doi:10.1039/D0FD00015A.

41. Seo, S. et al. Two Aluminophosphate Molecular Sieves Built from Pairs of Enantiomeric Structural Building Units. Angew. Chem. Int. Ed. 57, 3727-3732 (2018).

42. Foster, M. D. \& Treacy, M. M. A Database of Hypothetical Zeolite Structures: http://www.hypotheticalzeolites.net/ (accessed May 20, 2020).

43. Li, Y., Yu, J. \& Xu, R. Criteria for Zeolite Frameworks Realizable for Target Synthesis. Angew. Chem. Int. Ed. 52, 1673-1677 (2013).

44. Parise, J. B. Crystal Structures of Related Novel Aluminophosphate Frameworks: $\mathrm{AlPO}_{4}-21$ (py), $\mathrm{AlPO}_{4}-\mathrm{EN} 3$ (en) and A Structural Model for $\mathrm{AlPO}_{4}-25$. in Studies in Surface Science and Catalysis (eds. Držaj, B., Hočevar, S. \& Pejovnik, S.) vol. 24 271-278 (Elsevier, 1985).

45. Hartmann, M., Prakash, B. M. \& Kevan, and L. Multinuclear MAS NMR study on the microporous aluminophosphates AlPO4-41 and SAPO-41. J. Chem. Soc. Faraday Trans. 94, 723-727 (1998).

46. Yan, W. et al. [A112P13O52]3-[(CH2)6N4H3]3+: An Anionic Aluminophosphate Molecular Sieve with Brönsted Acidity. Chem. Mater. 12, 2517-2519 (2000).

47. Davis, M. E., Saldarriaga, C., Montes, C., Garces, J. \& Crowdert, C. A molecular sieve with eighteen-membered rings. Nature 331, 698-699 (1988).

48. Broach, R. W., Wilson, S. T. \& Kirchner, R. M. Corrected crystallographic tables and figure for as-synthesized AlPO4-14. Microporous Mesoporous Mater. 57, 211-214 (2003).

49. Jordá, J. L. et al. Structure analysis of the novel microporous aluminophosphate IST-1 using synchrotron powder diffraction data and HETCOR MAS NMR. Microporous Mesoporous Mater. 65, 43-57 (2003).

50. Pluth, J. J., Smith, J. V. \& Bennett, J. M. Microporous aluminophosphate number 17 with encapsulated piperidine, topological similarity to erionite. Acta Crystallogr. C 42, 283-286 (1986).

51. Josien, L., Simon, A., Gramlich, V. \& Patarin, J. Synthesis and Characterization of the New Hydroxygallophosphate Mu-18 with a Framework Topology Closely Related to That of the Hydroxyaluminophosphate AlPO4-EN3. Chem. Mater. 13, 1305-1311 (2001). 